This item was submitted to Loughborough's Research Repository by the author.

Items in Figshare are protected by copyright, with all rights reserved, unless otherwise indicated.

\title{
Social sustainability in design: moving the discussions forward
}

PLEASE CITE THE PUBLISHED VERSION

http://dx.doi.org/10.1080/14606925.2015.1059604

PUBLISHER

(c) Taylor \& Francis

VERSION

AM (Accepted Manuscript)

PUBLISHER STATEMENT

This work is made available according to the conditions of the Creative Commons Attribution-NonCommercialNoDerivatives 4.0 International (CC BY-NC-ND 4.0) licence. Full details of this licence are available at: https://creativecommons.org/licenses/by-nc-nd/4.0/

\section{LICENCE}

CC BY-NC-ND 4.0

\section{REPOSITORY RECORD}

McMahon, Muireann, and Tracy Bhamra. 2015. "Social Sustainability in Design: Moving the Discussions Forward". Loughborough University. https://hdl.handle.net/2134/18883. 


\section{Social Sustainability in Design: Moving the discussions forward.}

\section{Introduction}

The emphasis in sustainability, to date, has tended to be placed on economic and environmental issues, as these are tangible, measurable and in some respects the 'lowest hanging fruit' to address (Woodcraft et al., 2011). First published in a 1999 OECD expert workshop, and becoming more prevalent in the contemporary Sustainable Development [SD] debate, is the need to shift the balance between economics and environment to include more social and human concerns (OECD, 2000). Social sustainability, although not clearly defined or agreed upon, is often where all the indefinable elements of SD are placed (Findeli, 2008).

The majority of literature describes the social, often 'unquantifiable', elements of Sustainable Design to mean issues of health and safety, equity, human rights, social entrepreneurship, governance and corporate responsibility, consumption habits and efficiency (The Designers Accord, 2010). Lilley has distilled Social Sustainability to include the softer agendas of 'personal responsibility, equitable distribution of social capital, meeting basic needs, quality of life, health, well-being and happiness, democratic participation, trusting, harmonious and cooperative behaviour and preserving social and cultural dynamism' (Lilley, 2007, Lilley, 2009). This can be further expanded to include history, traditions, dialogue, equity in expressing ideas, cultural diversity, ethics, collective and personal responsibility, satisfying physical and social needs, compromise, accountability, self-fulfilment and altruism (McKenzie, 2004, Willard et al., 2010, Nagel et al., 2012, Woodcraft et al., 2011). Behind this 'laundry' list, however is the common belief in all descriptions of social sustainability, that the human being is tasked with facilitating the problem-solving process (Klein 2009).

Arguments and discussions around the classic Brundtland definition for Sustainability suggest that it offers no practical path forward or suggestions of how to achieve the balance between the three tenets comprising sustainability (Dewberry, 2011, McKenzie, 2004, Griggs et al., 2013). Difficulties arise as social issues tend to change more dramatically between locations, people and cultures, than do the environmental and economic issues (Casimir and Dutilh, 2003). Like the notion of social impacts in Sustainable Development, the idea of social sustainability in 
design often presents more problems than it resolves. Social and human behaviours are intangible, unpredictable and difficult to control, ergo, difficult to change (IDEO, 2008, OECD, 2000). Although recognised as a difficult but necessary task, the inclusion of social aspects in any agenda leads to immeasurable elements that resultantly warrant the title of 'wicked' problems. These complex challenges present an opportunity for design as the solutions for sustainability must be creative and innovative if they are to generate any impact. Fortunately, design can offer this creative platform as it is the convergence of science, technology and the arts (Sotamaa, 2009).

\section{Moving Forward}

Alongside the dearth of a definition there is also a lack of discussion on how to practically implement social sustainability in design. Within this relatively new field the emphasis must be on agreeing definitions and measurement in an effort to build understanding and consensus (McKenzie, 2004). Despite the growing interest in sustainability we have yet to map and identify the competencies needed to achieve it in any real way (Dilnot, 2009). Several authors have begun to identify the competencies for Sustainability education (Wiek et al., 2011, Wals, 2010, Barth et al., 2007). These however still lie in the realm of theoretical discourses and so the next steps must be to explore methods of bridging the gap between theory and practice.

\section{Identifying social sustainability in design through a Delphi study}

Given the lack of a consensual definition for social sustainability within design, there was an apparent need to connect the gaps in literature with a clear understanding of what social sustainability means to design whilst also identifying the competencies necessary to effectively implement it through design work. These answers needed to be generated by as wide a variety of voices and perspectives as possible, particularly those practising in the field (Clayton, 1997a). The logistics of gathering these varied opinions from experts around the globe however could prove to be a difficult and complex task. Fortunately, after exploring a variety of research 
methods the Delphi method ${ }^{1}$ was identified as an appropriate technique for harnessing international expertise without the need for travel (Cohen et al., 2000, Landeta, 2006, Martin et al., 2008).

By collating the written responses of a group of experts consensus can be achieved on an area or where there is incomplete knowledge (O'Neill et al., 2009, Skulmoski et al., 2007, Landeta, 2006, Wicklein, 1993). The Delphi method allows for a group of individuals, related by discipline, to collectively deal with and develop causal relationships in complex social problems (Linstone and Turoff, 2002). It is also useful when a problem doesn't 'lend itself to precise analytical techniques but can benefit from subjective judgements on a collective basis' (Ibid). It is seen as an efficient tool for problem-solving by gathering together data not accurately known or available (Skulmoski et al., 2007, O'Neill et al., 2009). Using an iterative process (comprising two to three rounds), the method amalgamates the perspectives of the expert panel under one combined outcome (O'Neill et al., 2009). Continuous feedback between each of the rounds ensures that a valid consensus can be agreed upon with which all the experts are comfortable (Landeta 2006).

\section{Planning the study}

The first step in planning the study was to compile a list of experts. This is arguably the most important step in the process, as the choice and participation of the experts determines the quality of the outcomes (Okoli and Pawlowski, 2004). A Knowledge Resource Nomination Worksheet [KRNW] was compiled (Miaskiewicz and Kozar, 2011) and the nominated experts were divided into three relevant fields.

A Academics (particularly in product/industrial design with expertise in Sustainability);

A NGO's (involved in Design for Social Impact and design projects);

A Design Practitioners (those working professionally in the design industry with a focus on Sustainability or Responsible Practice).

\footnotetext{
${ }^{1}$ The Delphi Method consists of a group of expert responding to a series of questionnaires over at least two rounds of questioning. In between each round the responses are collated, analysed and fed back to the panel by the researcher LINSTONE, H. A. \& TUROFF, M. (eds.) 2002. The Delphi Method: Technique \& Applications, Boston: Addison-Wesley Publishing.
} 
These categories were decided upon based on collating a panel of experts who could make an effective contribution to the development of a definition (O'Neill et al., 2009, Okoli and Pawlowski, 2004). The criterion for selection was based on the experience of the expert in the area of Sustainable Design (a minimum of five years) and their prior knowledge of Sustainability relating to one or more of the three fields. Because of this prior knowledge choosing a random selection of experts was not considered (O'Neill et al., 2009).

A small number of experts, known to the researcher, were contacted first and they were asked to nominate more experts, employing the 'snowball effect' (Skulmoski et al., 2007). An initial panel of thirty three experts were contacted (Clayton, 1997b). The experts spanned a diverse range of international locations to provide for a global spread of the panel. Throughout the study, the identity of each of the experts was kept anonymous to the other participants. This was recommended to avoid 'competition' or conflict and to ensure the responses were impartial and based on the individual's opinion, without influence from others (Hsu and Sandford, 2007).

\subsection{Ensuring success}

The success of the study was dependent on a number of factors: choosing the correct experts with relevant expertise and who are willing to take part; timing; communication and clarity of information. To ensure success the first round content was sent on the day the experts were asked to participate to ensure no time elapsed between agreement to participate and commencement of the study. The experts were also provided with a context for the study so they would know the reasons why the study was being conducted and why their expertise was requested.

Gaining commitment from the experts required being up front about the premise for the study and the time required from the panellists. In order to ensure participation from the highest number of invited experts a brief over-view of the project was presented and the commitment expected from them explained in detail (timings- length of the study, time between rounds, duration of the questionnaires etc.). Twenty one of those who were initially contacted completed the first round of questions; the 63\% (21 respondents from the initial 33) can be 
claimed as a highly successful response rate. Further stability in the results was achieved with 19 $(90 \%)$ of the original 21 experts completing the three rounds of the study.

Each round took approximately one-two months to complete, including collection, collation and analysis of the results. The entire study took 3 months from Round 1 to the final feedback; this according to Landeta is an average timeframe for Delphi studies (Landeta, 2006). If there was a non-response the experts were contacted again and following this second contact all of the experts responded (Hsu and Sanford 2007). Feedback was provided between each round of the study so the participants could revise their opinions and add more depending on the outcomes from the previous rounds. This ensured the experts were invested in every round of the study (Landeta 2006).

Prior to sending out the material for each round the questionnaires were pre-tested on a small sample to try to eliminate any potential issues that may occur e.g. ambiguous interpretations, information gaps or any syntax mistakes (Landeta, 2006). Email was used as the communication tool for the process, as this allowed for quick feedback and instant contact with the panellists irrespective of their locations. The iterative nature of the Delphi process, and the openness to deal with both consent and dissent added further rigour to the process and ensured researcher reflexivity was avoided. The researcher merely collating and analysed the results- the participants ultimately decided on the final statements.

\subsection{Dealing with the Data}

The qualitative data from the responses across Rounds 1 and 2 were collated, organised and then analysed using three levels of coding: Open (preliminary codes were generated following a review of the responses); Axial Coding (reorganising the original codes into child and parent codes) and finally Consolidated Coding from which the cohesive definitions and amalgamated responses were formed (Gray, 2009). The quantitative data was transferred onto spread-sheets and the means and frequencies of responses calculated ${ }^{2}$.

\footnotetext{
2 The full analysis process and detailed results can be found in MCMAHON, M. 2013. Designed from the inside out. $\mathrm{PhD}$, University of Loughborough.
} 


\section{Implementing the Study}

\subsection{Round 1}

Round 1 comprised a semi-structured questionnaire with two sections. The modified Delphi method encourages a semi structured format with a narrower scope so the experts time is used efficiently, any ambiguities can be avoided while ensuring the most relevant and important topics are being dealt with from the offset (O'Neill et al., 2009). The first section presented the experts with a list of 24 competencies, derived from the literature review. The experts were asked to:

1. Rate the competencies listed (using a Likert scale ${ }^{3}$ ), as you deem them to be important to Social Sustainability in design.

2. Rate your own degree of knowledge of the particular competency (high, medium or superficial). The experts were asked to do this as a means of self-assessment, where their answers could be used to weight their responses to the perceived importance of the competency (Landeta, 2006).

3. Provide any other competencies that you consider important (but are not on the original list).

An open ended question formed the second part of the questionnaire. The panel was asked to

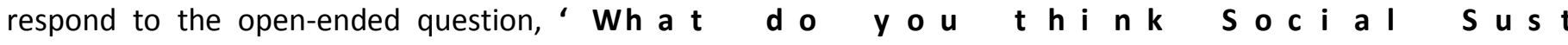
d e s i g n ?'

\subsection{Results of Round 1}

\subsubsection{Question 1}

On average the experts had a high to medium level of knowledge of the competencies. The experts rated those competencies of which they had the most knowledge to be the most relevant. Definite trends emerged in the ranking of competencies. Similar competencies were marked as relevant and the majority of experts viewed the same competencies as having little or no relevance. This meant that the irrelevant competencies could be eliminated which resulted in five competencies being eliminated from the list (Accountability, Confidence, Judgement, Comparison and Investment). Following feedback from the panel on Question 1 two further competencies, 'Humility' and 'Humour' were both added to the list for Round 2.The

\footnotetext{
${ }^{3}$ (1: Essential, 2: Desirable but not essential, 3: Slightly useful, 4: Irrelevant).
} 
revised list of competencies was arranged into a visual diagram to be used for Round 2's questionnaire (Figure 4).

Figure 1: Framework of competencies for Social Sustainability in design [Rev.1]

\subsubsection{Question 2: 'What does social sustainability mean to design?'}

The aim of Question 2 (open structure) was to find a common 'definition' for Social Sustainability for design that reflected the opinions and diversity of all of the experts. The responses from the experts were collated and coded (as described in 3.2). From this coding exercise a definition was extracted. This was revised and refined a number of times employing the various coding levels, and then sent for review by 2 independent experts to ensure clarity and coherency. It is important to note that although the definitions offered by the experts essentially dealt with the same topic the variety of voices and perspectives was mixed with each expert focusing their response on areas of importance to them and the context in which they worked. As such it was very important that each expert would hear his/her own voice in the definition as it moved closer toward consensus.

Figure 2: Social Sustainability in design construct [Rev.1]

\subsection{Issues arising from Round 1}

Feedback from the panel (through emails) stated that they found the approach, and the topic, interesting and relevant. A number of issues were highlighted during the analysis phase which was corroborated through the email feedback from the panel experts. These changes greatly reduced the drop-out rate during the remainder of the study (Hsu and Sandford, 2007). The four main issues that would impact on the subsequent round were:

1. Did people interpret competencies differently? Following comments on the Round 1 questionnaire some experts noted that it was difficult to complete the questionnaire, because there could be ambiguity in interpreting the competencies. Asking people to define what they meant, or understood, by the various competencies in the subsequent round would help to overcome this issue. 
2. What are the definitions for the most important competencies? How would the experts define the competencies and most importantly how would they manifest in a designer practice? These questions come from wanting to move from rhetoric to action.

3. Could the panel move closer to a consensual definition for Social Sustainability in design? In order to do this a working definition was provided to which the panel could respond, comment and provide feedback on. Some experts, following Round 1, commented that it would be more productive to have statements instead of openended questions. This way the feedback would be richer and more useful in moving towards consensus.

4. Were the voices of the experts all audible in the 'definition' for Social Sustainability in design? Were they satisfied with the definition and could they advocate it in their profession? Have the experts any additional comments on the 'definition' in order to enrich and refine it?

\subsection{Round 2}

\subsubsection{Structure of Round 2}

Round 2 served to clarify the issues arising from Round 1 and also to get feedback from the panel on the definition and framework of competencies collated from their responses. The questionnaire contained a brief background to remind the participants of the purpose of the study. The findings from Round 1 were explained, along with a short description of the methods used for collation and analysis of the responses. Providing feedback between rounds is a unique characteristic of the Delphi method and it serves to 'give back' to the panel (Clayton, 1997b, Landeta, 2006). This allowed the participants to understand the process and as a result, understand where and why decisions were made and from where the definition had come.

Firstly, the experts were asked to answer a few attitudinal questions to gauge their opinions on the working definition. By responding either 'Yes, No or Partially' to the following 'opinion' questions: Do you agree with this statement? Is your voice recognisable in the statement? Does the statement cover your views? a quick overview of the consensus on the definition was provided. The experts were then asked to comment on the working definition, thus providing the opportunity to revise or change their own previous statements, based on the general findings (Martin et al., 2008, Hsu and Sandford, 2007). 
In the Question 2 the experts were asked to choose three competencies from the list collated through Round 1. This choice was to be based on what the expert felt were the most important, relevant and realisable competencies within the context of educating for social sustainability in design. Observations from Round 1 highlighted that the experts' interpretation of the competency could vary hugely and unanimity needed to be sought to ensure the findings could be consensual. The experts were asked in Question 2 to briefly describe what they understood by the competency. It was hoped that at least one definition would be offered for each competency. However there was no way of ensuring this as the questionnaire was not intended to force the experts to define specific competencies, instead they had to choose the ones they felt most relevant.

\subsubsection{Analysis of data from Round 2}

\subsubsection{Question 1}

With regard to the statement generated in Round 1, the response was predominantly positive. Figure 3 below shows the responses to the three attitudinal questions.

Figure 3: Expert responses on social sustainability statement from Round 1 (1=Yes; $2=$ Partially; $3=$ No).

Following the initial analysis (quantitative), it was decided to retain the main meaning and intent of the statement and to review the open-ended comments as well as the email feedback, and to make changes based on these. The feedback also indicated that the statement wasn't in fact a definition as it couldn't be taken as 'definitive', given the small sample size. One expert suggested re-terming the statement as a Construct, on which to base the current interpretation and understanding of social sustainability.

In their feedback some experts noted that the construct was pragmatic, others said it wasn't pragmatic enough (needing more action-oriented language) 'Design is not (only) characterised by a final result, but also by the process how to get there' (Expert 9). It is important to note that agreement and disagreement are equally important at every stage of the feedback loop (O'Neill et al., 2009). In order to find this balance the language of the entire construct was re-focused to be more 'doing' and action-oriented, moving away from abstract language. 
It was important to recognise that the construct cannot solve every issue presented through the extremely complex and wicked problems associated with social sustainability. "I think there is a danger of design by committee (remember the camel), that is the definition is trying to encompass everything. Maybe the questionnaire could be used not to define social sustainability but to inform your viewpoint' (Expert 13). Another expert noted that the definition might not be ambitious enough 'While designers are not solely responsible, they should overestimate the role they can play... (Designers are supposed to be mavericks, divergent thinkers, leaders, innovators and change makers)' (Expert 14). A compromise needed to be found between being too ambitious and not recognising the important role the designer can play. The construct should provide designers with a way to begin to change their own practices and help influence others to do similar, thus moving the sustainable design agenda forwards. As such the construct would need to differentiate between socially sustainable design (the outcomes) and socially sustainable designing (the process). This would enable designers to make informed decisions as to where their views and individual practices lie within the sustainable design debate.

The last line of the statement was cause for comment 'Above all design must create fun, enjoyable and enriching experiences for PEOPLE'. One expert observed that this might be reducing the practice of sustainability to too playful a role and as such diminish the potential impact '...design is more than an experiential tool and while social impact design does need to be user-centric, there are many functions and purposes it fulfils that may have to do with systems change that go beyond the relationship with people' (Expert 9). Also this statement, according to one respondent, was too anthropocentric again continuing '... to put HUMAN needs at the forefrom [sic]' (Expert 4). As a result the statement was revised to still include the 'humour' element, but to be less flippant. The final construct (Figure 6) was devised from the collation, analysis and interpretation of the expert panel responses.

Figure 4: Social Sustainability in design construct [Rev.2]

\subsubsection{Question 2}


From the responses to the competencies in Question 2 a clear trend was observed that rather than just defining the competency, the expert panel gave real, practical and considered ways to resolve the issues or to implement the competency. It was interesting to note that the competencies the experts chose, while they did correlate to the hierarchical ranking in Round 1, there were a number of competencies that had been ranked low in the previous round but were defined by over $25 \%$ of the experts. For example Empathy was ranked $7^{\text {th }}$ in Round 1 , yet over $33 \%$ of the experts defined it in Round 2 and Engagement was chosen and defined by $28 \%$ (5) of the experts but it has ranked $23^{\text {rd }}$ in Round 1.

Because the definitions went beyond rhetoric the experts began suggesting action in their language and content. One expert offered that the competencies could be used to move the practice of sustainable design forward 'I find the key themes (and sub words) very interesting and could build on those as a design language to shift the paradigm of the single bottom line' (Expert 8).

Finally the competencies were arranged into a visual graphic to explain the connections between them. Three overarching competencies emerged from the definitions: Critical Thinking (green in Figure 5); Team-work (orange in Figure 5) and Social Interaction (blue in Figure 5). Certain competencies crossed over the three areas- these can be seen with two or three different outlines. New Processes, New Perspectives and Humour don't fall specifically into any one category and, as such, are categorised as overarching competencies that should be prevalent in all aspects.

Figure 5: Framework of competencies for Social Sustainability in design [Rev.2]

\subsection{Round 3}

Following the analysis of the answers from the previous round the results were compiled into the final framework of competencies deemed relevant to the development of social sustainability skills and capacities in design as illustrated in Figure 5 above. The definition of 
social sustainability was re-categorised as a construct following the analysis phase as well as comments from the panel.

Both the construct and the framework were sent to the panel one more time for comment and feedback. The entire expert panel responded to the mail and the feedback was extremely positive for the final stage of the study. One expert noted that the dialogue and debate sparked amongst the panel might prove more valuable than the actual responses '...that is do you need tidy definitions if you can get students and designers to consider the questions?' [Email from Expert 13, 22.05.2012]. In the previous two stages, these discussions (between the expert and the researcher), which were conducted via email, were included anecdotally when the responses were being analysed. This led to the interpretation of the data stemming, not just from the data itself, but from the comments and observations that accompanied the responses. Richer results emerged as a consequence of the unsolicited additional input from the expert panel.

\section{Discussion}

\subsection{The Delphi Method}

The Delphi method proved to be an extremely useful tool in this instance because it allowed access to a wide variety of design experts dispersed around the world to gather information which spanned diverse perspectives and opinions. It proved challenging at times dealing with the diversity as explanations often got 'lost in translation' across email and distance. This was particularly evident when language differences came into play. Fortunately, due to establishment of open communication channels from the offset, any issues were resolved quickly with follow-on emails and phone-calls where necessary.

A number of other positives of employing the Delphi technique emerged during each round, these included:

A 'Secrecy' the anonymity aspect allowed the experts to be upfront and honest with their feedback. 
A The time between rounds allowed the participants to reflect on the topic and their opinions on the issues being raised.

A The feedback loops ensured the experts could see their ideas being built upon and integrated into collective constructs and ideas (Miaskiewicz and Kozar, 2011)

Perhaps the most interesting outcome of conducting the study was the enthusiasm with which the panel took part and how open and generous they were in sharing their knowledge, time and experience. Figure 6 below draws together some of the rich feedback offered by the experts over the three rounds. This evidences that there is a huge wealth of knowledge on the area of Social Sustainability that needs to be drawn together; designers just need to be given the opportunity to formulate and offer their thoughts in an open and dialogical platform.

\section{Figure 6: Expert Panel Feedback}

Despite the perspectives of the expert panel being diverse they tended to agree on the bigger issues e.g. that the constructs for social sustainability and the competencies needed to be pragmatic and action-oriented and that the definition for social sustainability in design couldn't be a definition. The experts often gave information above what was asked of them, this added to the richness of each round and informed the subsequent rounds. This indicates that the topic is relevant and necessary as the participants felt they had additional information to offer; which in turn enriched and expanded the discourse generated through the Delphi beyond initial expectations.

In order to build the data from the Delphi study into a usable format, both the construct for social sustainability and the constructs for the key competencies offered by the expert panel, required further clarification.

\subsection{Social Sustainability Construct}

Undertaking the Delphi highlighted that it was too difficult (and perhaps unnecessary) to form a consensual 'definition' for social sustainability in design. It is too complex an issue that one 
definition could not be sufficient. As such, a construct is far more useful for the purposes of moving research forward (McKenzie, 2004).

From a practical perspective the construct needed to be built into a more useful format that could be employed as a foundation for future design work. This way the construct would help designers and design educators to adopt a sustainability lens through which to view potential themes and briefs. In order to do this a set of codes or organisational themes were developed from Wiek et al.'s article (2011) and distilled into Table 5 and then layered over the final construct (Figure 7).

Table 1: Codes for Analysis of Social Sustainability in design construct [devised from (Wiek et al., 2011)].

\begin{tabular}{|c|l|l|}
\hline 1 & \multicolumn{1}{|c|}{ Code } & \multicolumn{1}{c|}{ Sub Code } \\
\hline 2 & Ability to Think & To think in the big picture, complex ideas, systems-thinking \\
\hline 3 & $\begin{array}{l}\text { Ability to understand/ } \\
\text { interpret }\end{array}$ & $\begin{array}{l}\text { To take the abstract ideas, navigate them, reconcile them, } \\
\text { negotiate the complexity and to create visions for } \\
\text { sustainable solutions. }\end{array}$ \\
\hline 4 & Ability to implement & $\begin{array}{l}\text { To understand complicated ideas and to draw together } \\
\text { disparate strands and perspectives. Intimate understanding of } \\
\text { complex systems. }\end{array}$ \\
\hline 5 & Ability to Do & $\begin{array}{l}\text { To put the understanding of the issues into action. Crossover } \\
\text { with 'Do', however, do is the real brass tacks of putting it } \\
\text { into action. Implement is the broader sense of getting the } \\
\text { preparation done and bringing about solutions. }\end{array}$ \\
\hline 6 & $\begin{array}{l}\text { The time for talk is over! To put the rhetoric into action. To } \\
\text { really 'do'-get stuck in and make an effort to solve some of } \\
\text { the problems even if it isn't entirely successful. }\end{array}$ \\
\hline $\begin{array}{l}\text { To collectively implement ideas, being able to marry the } \\
\text { sophistication of collaboration with bringing about change. } \\
\text { To be able to facilitate, participate, lead, have empathy, } \\
\text { manage stakeholder collaboration. }\end{array}$
\end{tabular}

Figure 7: Social Sustainability in design construct [Rev.3] 
With this coded construct, words and phrases can be selected and used as pragmatic building blocks for the development and exploration of project briefs. In an educational context, learning outcomes can be linked to these words/phrases, in order to allow participants to explore and develop the core competencies associated with them. In a professional capacity, designers can use the phrases/words to affect decision-making and idea selection throughout the design process.

\subsection{The Framework of key competencies for social sustainable in design}

The competencies, that enable designers to address the complex challenges presented by sustainability, were determined through the literature review and revised by the panel of expert respondents in the Delphi Study. The descriptions emanating from the study had to lead to something practical and useful for future projects; similar to the construct. Following analysis it was clear that the competencies needed to be connected together as they are not stand alone skills and capacities. Collating the results from Round 1 and Round 2 we can see the emergence of relationships between the competencies (Figures 4 and 5). Connections between the competencies are embedded with both explicit and implicit links that involve personal and interpersonal behaviours (Wiek et al., 2011). Some cannot exist without others and some follow as consequence of others (Figure 8).

The results from the final Round of the Delphi study were reviewed and collated into a number of descriptions or constructs. Keywords were highlighted in each description; these words had an action orientated focus, in-keeping with the pragmatic nature of the Social Sustainability construct. These words were combined and cross-over eliminated.

The combined descriptions were collated and a series of questions were generated under each competency that would enable designers to frame project briefs and evaluate design outcomes. In order to refine the framework, various measures for evaluating similar and related competencies were reviewed ${ }^{4}$.

\footnotetext{
${ }^{4}$ Wilder Collaboration Factors MATTESSICH, P. W., MURRAY-CLOSE, M. \& MONSEY, B. R. (eds.) 2001. Collaboration: What makes it work: Amherst H. Wilder Foundation.; Watson Glaser Critical Thinking Appraisal; Critical Thinking Assessments ENNIS, R. H. 1985. A logical basis for measuring critical thinking skills. Educational Leadership, STERNBERG, R. J. \& BARON, J. B. Ibid.A state-wide approach
} 
This is not a comprehensive (nor is it intended to be a complete) list; generating a definitive list of the competencies is an impossibility (Barth et al., 2007). Given the complexity and the everchanging nature of social issues, the framework is constantly evolving in a similar way to the construct. Thus, the most relevant and realisable competencies, within the context of current design practice, have been identified. Examining and refining the Framework, re-iterated that all the competencies for social sustainability in design can't be developed or encouraged in isolation. Each competency impacts on others and cannot occur without interaction and dialogue between participants in the collaboration. Figure 8 below illustrates the connections between the competencies under the three umbrella competencies of Communication, Interaction and Critical Questioning. Similar to the arrangement in Figure 5 a number of the competencies in Figure 8 fall under one or more of the umbrella competencies with two way exchanges running between them.

These interdependencies between the competencies force the recognition that they cannot be cultivated through a stand-alone educational project. Instead projects should brick on top of each other and increase in complexity incrementally. Resultantly the emergence of the competencies happens over a longer period of time, avoiding confusion or complication, and leads to a more lasting and transformative experience.

Figure 8: Framework of competencies for Social Sustainability in design [Rev.3]

\subsection{Practical Applications for Design}

The reality of developing the competencies is demonstrably more complex than 'doing' a design project, fulfilling a brief or listing a set of desired learner characteristics. For all, or some, of the competencies to be attained to any level, designers must be given the correct contexts and opportunities for restructuring old, and acquiring new, knowledge (McMahon, 2013). The

to measuring critical thinking skills, ENNIS, R. H. An outline of goals for a critical thinking curriculum and its assessment. Sixth International Conference on Thinking 1994 MIT, Cambridge MA, ENNIS, R. 1993. Critical Thinking assessment. Theory into Practice, 32.; Higher Order Learning Rubrics PIERCE, W. Designing Rubrics for assessing higher order thinking. AFACCT, 2006 Columbia, MD.; Facione's Core Skills of Critical Thinking (Facione 2011); Sense-making (Dervin 1992, UNIVERSITY OF TWENTE. 2012. Sense-making [Online]. Available:

http://www.utwente.nl/cw/theorieenoverzicht/Theory\%20Clusters/Organizational\%20Communication/Sensemaking.doc/ [Accessed 30.01.2012.; Valencia College Core Competencies VALENCIA COMMUNITY COLLEGE. 2011. Valencia Core Competencies [Online]. Available: http://valenciacollege.edu/competencies/ [Accessed 27.06.2011, VALENCIA COMMUNITY COLLEGE 2006. Measuring my Critical thinking.; Blooms Taxonomy of Learning Domains (Clark 2011) and Hofstede's views on culture SCHADEWITZ, N. 2009. Design pattern for cross-cultural collaboration. International Journal of Design, 3, 37-53. 
competencies emerged when designers are exposed to new and different perspectives, and are challenged to navigate through the diverse opinions and processes these perspectives present.

As discussed by Barth et al. (2007) the competencies cannot be taught instead they are learned. This learning can happen through real-life experiences that offer exposure to certain conditions such as diverse environments, industrial practice, people and cultures etc. (Brundiers et al., 2010). The practical applications of both the construct and the competencies, beyond moving the debate forward, lie in their usefulness for building project briefs and evaluating design outcomes. This would be particularly useful education, as learning outcomes for projects can be built around the competency development and educators can explore how design students develop the competencies over time. Professionals can use the construct in particular to guide design directions and develop criteria to raise client awareness about sustainability issues.

\section{Conclusions}

Compiling the Delphi findings raised a number of interesting issues. While the majority of the experts agreed that there needed to be a base for social sustainability in design, some argued that current perspectives were too anthropocentric and were neither pragmatic nor measurable. The literature, unfortunately, further highlights that the challenge for design has changed over the past number of years, with the negative impact of current consumption and production practices coming to the fore (Dewberry, 2011, Tischner, 2008). The outcomes from the Delphi Study have gone a way towards dispelling the some of the ambiguity and the iterative nature of the method itself ensured the results underwent several rounds of refinement based on the formal (questionnaires) and informal (conversations between the experts and the researcher) feedback. This rigorous approach made certain that the construct and framework are expressed in practical language with which designers are both familiar and comfortable. The construct aims to move beyond mere definition and into identifying key characteristics and 'the precise causal relationships between its various aspects' (McKenzie, 2004).

This research does not purport to present a definitive description of social sustainability or the competencies necessary for its implementation in design; rather it is offering working constructs that could be applied practically in the generation of design projects and also in the evaluation 
of design solutions. It is a snapshot in time that can be adjusted, built upon and modified as the debate evolves and matures. This could go some way towards easing the complexity surrounding social issues that combine with environmental and economic competencies under the sustainable triple bottom line. However, if we de-couple the three tenets of sustainability and define them as separate agendas then, it could be argued, that we are countering the overall supposition of sustainable development, in that it is supposedly a holistic approach to addressing the impact of negative human behaviour. On this premise, the construct for social sustainability, alongside the Framework of competencies, presented in this paper, layers on top of the existing definition of Sustainable Design in an effort to move the agenda forward and open dialogical channels for discussion and debate. These discussions will encourage designers to examine the confluences of social, environmental and economic issues and within this exploration uncover the potential opportunities for design innovations it presents.

There is an apparent opportunity for designers, in both professional practice and in education, to use the design based Construct and Framework to underpin design briefs and evaluate ideas and concepts over the entire design process. Given that both are framed in action-oriented, dynamic and positive language it corroborates that Sustainable Design is not a barrier to design innovation, in fact it is a vehicle by which to implement it.

\section{References}

BARTH, M., GODEMANN, J., RIECKMANN, M. \& STOLTENBERG, U. 2007. Developing key competencies for sustainable development in higher education. International Journal of Sustainability in Higher Education, 8, 416-430.

BRUNDIERS, K., WIEK, A. \& REDMAN, C. L. 2010. Real-world learning opportunities in sustainability: from classroom into the real world. International Journal of Sustainability in Higher Education, 11, 308-324.

CASIMIR, G. \& DUTILH, C. 2003. Sustainability: a gender studies perspective. International Journal of Consumer Studies, 27, 316-325.

CLAYTON, M. 1997a. Delphi: a techniqie to harness expert opinion for critical decisionmaking tasks in education. Educational Psychology, 17, 373-386.

CLAYTON, M. J. 1997b. Delphi: a technique to harness expert opinion for critical decision-making tasks in education. Educational Psychology, 17, 373-386.

COHEN, L., MANION, L. \& MORRISON, K. 2000. Research Methods in Education, London \& New York, Routledge Falmer. 
DEWBERRY, E. 2011. Developing an ecology of mind in design. In: SOCIETY, T. D. (ed.) 18th International Conference on Engineering Design (ICED 11). Copenhagen.

DILNOT, C. 2009. Sustainability as a project of history. In: PREDAN, B. \& POZAR, C. (eds.) Sustainable alternatives in design. Ljubljana: The Architecture Museum of Ljubljana.

ENNIS, R. 1993. Critical Thinking assessment. Theory into Practice, 32.

ENNIS, R. H. 1985. A logical basis for measuring critical thinking skills. Educational Leadership.

ENNIS, R. H. An outline of goals for a critical thinking curriculum and its assessment. Sixth International Conference on Thinking 1994 MIT, Cambridge MA.

FINDELI, A. 2008. Sustainable Design: A critique of the current tripolar model. The Design Journal, 11, 301-322.

GRAY, D. E. 2009. Doing research in the real world, London, SAGE Publications Ltd.

GRIGGS, D., STAFFORD-SMITH, M., GAFFNEY, O., ROCKSTROM, J., OHMAN, M. C., SHYAMSUNDAR, P., STEFFEN, W., GLASER, G., KANIE, N. \& NOBLE, I. 2013. Policy: Sustainable development goals for people and planet. Nature, 495, 305-307.

HSU, C.-C. \& SANDFORD, B. A. 2007. Minimizing non-response in the Delphi process: How to respond to non-response. Practical Assessment, Research and Evaluation, 12.

IDEO 2008. Aligned for Sustainable Design: An A-B-C-D approach to making better products. In: IDEO (ed.). Business for Social Responsibility.

LANDETA, J. 2006. Current validity of the Delphi method in social sciences. Technological Forecasting and Social Change, 73, 467-482.

LILLEY, D. 2007. Designing for behavioural change: reducing the social impacts of product use through design. Doctoral Thesis, Loughborough University

LILLEY, D. 2009. Design for sustainable behaviour: strategies and perceptions. Design Studies, 30, 704-720.

LINSTONE, H. A. \& TUROFF, M. (eds.) 2002. The Delphi Method: Technique \& Applications, Boston: Addison-Wesley Publishing.

MARTIN, J. L., NORRIS, B. J., MURPHY, E. \& CROWE, J. A. 2008. Medical device development: The challenge for ergonomics. Applied Ergonomics, 39, 271-283.

MATTESSICH, P. W., MURRAY-CLOSE, M. \& MONSEY, B. R. (eds.) 2001. Collaboration: What makes it work: Amherst $\mathrm{H}$. Wilder Foundation.

MCKENZIE, S. 2004. Social Sustainability: towards some definitions. Hawke Research Institute Working Papers Series [Online]. [Accessed 03.02.2012].

MCMAHON, M. 2013. Designed from the inside out. PhD, University of Loughborough.

MIASKIEWICZ, T. \& KOZAR, K. A. 2011. Personas and user-centered design: how can personas benefit product design processes? Design Studies, 32, 417-430.

NAGEL, R. L., PAPAPAS, E. C. \& PIERRAKOS, O. 2012. On a vision to educating students in sustainability and design- The James Madison University School of Engineering approach. Sustainability, 4, 72-91.

O'NEILL, S., MURRAY, S. \& CONBOY, K. 2009. A Delphi study on collaborative learning in distance education. 17th European Conference on Information Systems. Verona, Italy. 
OECD 2000. Frameworks to Measure Sustainable Development. OECD.

OKOLI, C. \& PAWLOWSKI, S. D. 2004. The Delphi method as a research tool: an example, design considerations and applications. Information \& Management, 42, 15-29.

PIERCE, W. Designing Rubrics for assessing higher order thinking. AFACCT, 2006 Columbia, MD.

SCHADEWITZ, N. 2009. Design pattern for cross-cultural collaboration. International Journal of Design, 3, 37-53.

SKULMOSKI, G. J., HARTMAN, F. T. \& KRAHN, J. 2007. The Delphi method for graduate research. Journal of Information Technology Education, 6, 1-21.

SOTAMAA, Y. 2009. The Kyoto Design Declaration: Building a Sustainable Future. Design Issues, 25, 51-53.

STERNBERG, R. J. \& BARON, J. B. 1985. A state-wide approach to measuring critical thinking skills. Educational Leadership.

THE DESIGNERS ACCORD. 2010. Integrating sustainability into design education: The Toolkit [Online]. Available: http://edutoolkit.designersaccord.org/ [Accessed 30.10.2011 2011].

TISCHNER, U. 2008. Design for (social) sustainability and radical change. In: TUKKER, A. (ed.) Perspectives on radical changes to sustainable consumption and production. Greenleaf Publishing.

UNIVERSITY OF TWENTE. 2012. Sense-making [Online]. Available: http://www.utwente.nl/cw/theorieenoverzicht/Theory\%20Clusters/Organizatio nal\%20Communication/Sensemaking.doc/ [Accessed 30.01.2012.

VALENCIA COMMUNITY COLLEGE 2006. Measuring my Critical thinking.

VALENCIA COMMUNITY COLLEGE. 2011. Valencia Core Competencies [Online]. Available: http://valenciacollege.edu/competencies/ [Accessed 27.06.2011.

WALS, A. E. J. 2010. Mirroring, Gestaltswitching and transformative social learning: Stepping stones for developing sustainability competence. International Journal of Sustainability in Higher Education, 11, 380-390.

WICKLEIN, R. C. 1993. Identifying critical issues and problems in Technology Education using a modified Delphi technique. Journal of Technology Education, 5, 54-71.

WIEK, A., WITHYCOMBE, L. \& REDMAN, C. L. 2011. Key competencies in sustainability: a reference framework for academic program development. Integrated Research System for Sustainability Science.

WILLARD, M., WIEDMEYER, C., FLINT, R. W. \& WEEDON, J. S. 2010. The Sustainability Professional: 2010 Competency survey report. International Society of Sustainability Professionals.

WOODCRAFT, S., HACKETT, T. \& CAISTOR-ARENDAR, L. 2011. Design for Social Sustainability: A framework for creating thriving new communities. London: The Young Foundation. 